\title{
The Trend is Your Friend: A Note on An Ensemble Learning Approach to Finding It
}

\author{
Tzu-Pu Chang ${ }^{1}$, Yu-Cheng Chang $^{1}$ and Po-Ching Chou ${ }^{1}$
}

\begin{abstract}
The essential goal of trend-following investing is to precisely identify where the uptrend and downtrend are located. This paper thus provides a two-layer stacking technique, which is a novel ensemble learning approach, to predict such trends for the Taiwan Top 50 ETF. The proposed stacking technique stacks the predictors of support vector machine (SVM), multi-layer perception (MLP), adaptive boosting (Adaboost), and extreme gradient boosting (Xgboost), presenting empirical results whereby following the trends obtained from the stacking technique can generate positive returns and beat both conventional moving-average crossover and buy-andhold strategies.
\end{abstract}

JEL classification numbers: C02; G11

Keywords: Trend-following investing; Stacking technique; Ensemble learning; Machine learning; Taiwan Top 50 ETF

\section{Introduction}

An old adage says, "The trend is your friend, until the end when it bends." Trend-following investing is a well-known investment style that both institutional and retail investors would like to execute. The literature provides several pieces of evidence for the profitability of trend-following investing even after taking off the corresponding transaction costs and fees (Clare et al., 2016; Hurst et al., 2017). Undoubtedly, the key to success is to precisely identify (or predict) where an uptrend and downtrend occur. Two popular approaches in practice are to apply moving-average crossover and time-series momentum signals (Faber, 2007; Levine and Pedersen, 2016). Levine and Pedersen (2016) also prove that these two trend indicators are highly correlated. Over the past few decades, Raj and Thurston (1996), Papadamou and Tsopoglou (2001), and Neely et al. (2014) indicate that moving-average crossover and time-series momentum signals are profitable on different markets, such as foreign exchange, futures, and stock markets.

Instead of those popular methods mentioned above, this paper adopts an ensemble learning approach, which is a novel technique in terms of machine learning. In fact, over the past decades the theories and applications of machine learning have become some of the most popular research topics. With respect to investing issues, Huang et al. (2005) apply a support vector machine (SVM) approach to forecast the weekly return of the NIKKEI 225 index and conclude that the SVM model is better than the random walk, linear discriminant analysis, and quadratic discriminant analysis. Colombo et al. (2019) also use a SVM model to execute directional forecasts for carry trades and present that the performance of carry trades

\footnotetext{
${ }^{1}$ Department of Finance, National Yunlin University of Science and Technology, Taiwan
}

Article Info: Received: October 24, 2021. Revised: November 30, 2021. Published online: January 3, 2022 
improves substantially. Henrique et al. (2019) provide a complete review about financial market prediction by using machine learning algorithms.

Ensemble learning combines multiple single classifiers/algorithms to obtain a better predictive model with higher accuracy. Tsai et al. (2011) apply a heterogeneous ensemble learning, combining multi-layer perception (MLP), decision trees, and logistic regression, to predict quarterly returns of Taiwan's electronics index. ${ }^{2} \mathrm{Up}$ to now, there are three advanced and commonly-used ensemble techniques: bootstrap aggregating (bagging), boosting, and stacking (Nti et al., 2020). Using four market indices (U.S.A., India, Ghana, and South Africa), Nti et al. (2020) suggest that the stacking method (heterogeneous ensemble classifier) performs better than bagging and boosting (homogeneous ensemble classifier).

This study aims to identify and predict the trend of the Taiwan Top 50 ETF by applying a stacking ensemble technique. It looks to contribute to the literature in two ways. First, most studies in the literature predict the direction of future price movement with respect to the classification task. The direction movement is defined by stock price(s) going up/down after a particular period, such as daily direction, 30days-ahead, or one-year-head (Tsai et al., 2011; Ballings et al., 2015; Nti et al., 2020). Unlike the literature, this paper first identifies the turning points of stock prices and then finds where upward and downward trends are. Hence, this study aims to predict whether a stock price belongs to an upward or downward market one-day-ahead framework. Second, the stacking ensemble technique is rarely applied in the field of financial research. Thus, we adopt this technique and expect that the stacking ensemble technique can beat several methods, such as single classifiers, moving-average method, and buy-and-hold strategy.

Subsequent sections are arranged as follows. Section 2 presents the empirical data and methodology. Section 3 demonstrates the empirical results. Section 4 concludes this paper.

\section{Data and Methodology}

This paper selects the Taiwan Top $50 \mathrm{ETF}$ as the research object, because this ETF is the first one to be listed and the largest ETF in Taiwan. Moreover, the components of the Taiwan Top 50 ETF are the 50 largest listed companies in the Taiwan Stock Exchange. Thus, the Taiwan Top 50 ETF highly correlates to the market index - Taiwan Stock Exchange Capitalization Weighted Stock Index (TAIEX). The research period covers from 5/5/2004 to 12/11/2018 with a total number of 3,619 trading days. We separate this data into a training dataset (the first $85 \%$ of observations) and a testing dataset, respectively. Figure 1 presents the price series of the Taiwan Top 50 ETF covering the whole research period, in which the lowest price is NT\$19.33 per share during the 2008 global financial crisis and the highest price is NT $\$ 79.75$ at $8 / 30 / 2018$.

\footnotetext{
${ }^{2}$ Tsai et al. (2011) define heterogeneous ensemble learning as the combination of different types of classifiers or algorithms and use a majority voting method to combine the results coming from three classifiers.
} 


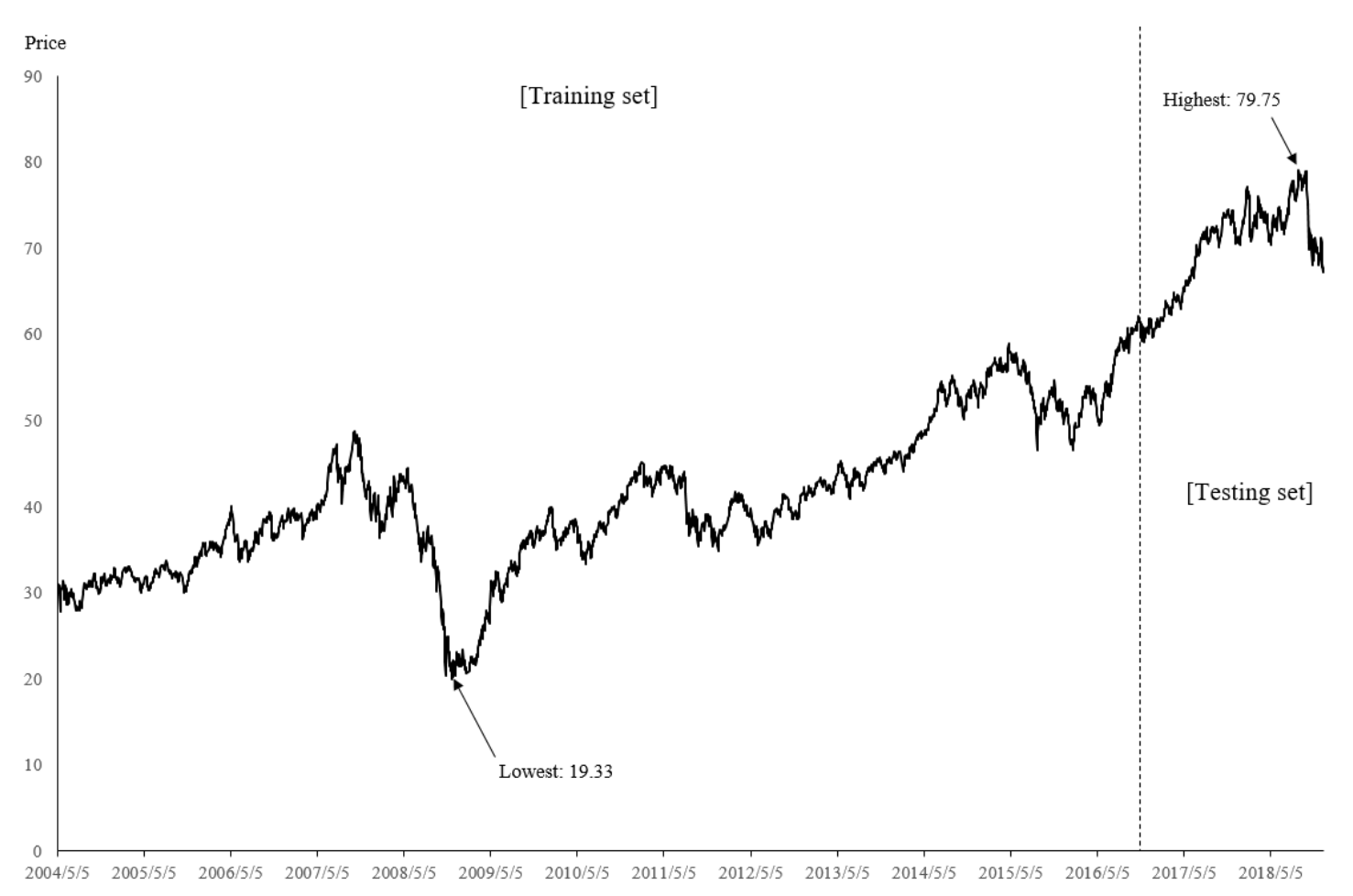

Figure 1: The daily closing price of the Taiwan Top 50 ETF

The target variable is a dichotomous variable defining that each trading day is classified as moving along an upward or downward trend. Therefore, how to identify upward and downward markets is a crucial task in this study. We follow a non-parametric method proposed by Chen (2009), in which he suggests a two-step method. First, on the basis of daily frequency, a peak in time $t$ is identified if its price satisfies $\left\{p_{t}>p_{t \pm n}\right\}$. It means that the price at a peak should be higher than that before and after $n$ days. Hence, a trough in time $t$ is defined if the price satisfies $\left\{p_{t}<p_{t \pm n}\right\}$. Second, after confirming each turning point of a stock price, an upward (downward) trend is determined from a certain trough (peak) to a successive peak (trough). This two-step method is also applied in the studies of Nyberg (2013) and Duong et al. (2021).

The input features include opening, high, low, and closing prices, volume, and twenty technical indicators for each day. Based on a multi-national survey from fund managers, Menkhoff (2010) presents that technical analysis is the most important technique for fund managers to determine future movements of stocks. We thus heavily rely on technical indicators to predict upward and downward trends. We also collect fourteen input features related to institutional and retail investors' trading/holding information, such as margin purchase, short sale, and net buy/sell of institutional investors. To incorporate the impact of international markets, this paper considers whether the NASDAQ index is in a bull or bear market in the previous day based on the same method mentioned above. ${ }^{3}$ Notice that all input features are standardized and lagged one day in this study.

With respect to the ensemble learning approach, this paper adopts a two-layer stacking ensemble technique. A general form for the two-layer stacking technique can be defined as:

\footnotetext{
${ }^{3}$ The full list of input features is available upon request to the author. The NASDAQ index is chosen, because most of the Taiwan Top 50 ETF constituents are related to technology stocks.
} 


$$
f_{S}(x)=\sum_{i=1}^{n} \alpha_{i} f_{i}(x)
$$

where $x$ is a set of input features; $f_{i}(x)$ denotes the predictions made from the $i^{\text {th }}$ base-learner; $f_{s}(x)$ denotes the stacked final prediction; and $\alpha_{i}$ is the weight for the $i^{\text {th }}$ base-learner, which is determined by the meta-learner. For the first layer (base-learner), we apply four base models: support vector machine (SVM), multilayer perceptron (MLP), adaptive boosting (Adaboost), and extreme gradient boosting (Xgboost). For the second layer (meta-learner), we adopt a decision tree classifier (i.e., CART algorithm) to train the predictions obtained from the four base models mentioned above. These classifiers are soundly established and commonly used in terms of stock market prediction (Ballings et al., 2015; Colombo et al., 2019; Henrique et al., 2019).

In order to examine the performance of the stacking technique, this paper further constructs a trading strategy and compares the corresponding profitability with four single classifiers, moving-average method, and buy-and-hold strategy. Recall that this paper aims to predict whether each trading day is classified into an upward or downward market. We take a long (short) position with the opening price at $t+1$ when we use all data available at $t$ to predict a downward (upward) market turning into an upward (downward) market at $t+1$. We also set a trading cost of $0.5 \%$ for each trade that is higher than the trading cost in reality $(0.385 \%){ }^{4}$

\section{Empirical Results}

This section only focuses on the performance of the stacking technique for the testing data. The opening price on the first day and the closing price on the last day in the testing data are NT\$60.69 and NT\$67.75 per share, respectively. Hence, the return of a buy-and-hold strategy is $11.1 \%$, which is a benchmark with respect to profitability comparison.

Table 1 presents the performance summary of four base-learners. As shown in Table 1, the prediction accuracy of all four base-learners is over 60\%. Among them, Adaboost and Xgboost have a higher accuracy of nearly 70\%. Unsurprisingly, both Adaboost and Xgboost are homogeneous ensemble classifiers, such that they can make a better prediction than the other two single classifiers. However, none of these base-learners are profitable, and two of them suffer negative total returns at about $-30 \%$ over the testing period, indicating that high prediction accuracy cannot ensure trading profitability. The MLP classifier (-10.20\%) performs better than the other three base-learners, implying that an overtrading problem causes higher trading costs and lower total returns as well as percent profitable. Maximum drawdown (MDD) denotes the maximum observed loss from a certain peak to a successive trough of an equity curve. If we buy one trading unit (1,000 shares), then these base-learners' MDDs are about 20\%, indicating that the base-learners' performances are risky and unstable.

Table 1: Performance indicators of the four base-learners

\begin{tabular}{lllll}
\hline Classifier & SVM & MLP & Adaboost & Xgboost \\
\hline Prediction accuracy & $60.00 \%$ & $65.30 \%$ & $68.60 \%$ & $68.40 \%$ \\
Number of trades & 73 & 35 & 65 & 76 \\
Total return & $-31.38 \%$ & $-10.20 \%$ & $-30.37 \%$ & $-24.21 \%$ \\
Average return & $-0.51 \%$ & $-0.31 \%$ & $-0.56 \%$ & $-0.36 \%$ \\
Percent profitable & $30.14 \%$ & $31.43 \%$ & $30.77 \%$ & $30.26 \%$ \\
Maximum drawdown & NT $\$ 13,584$ & NT $\$ 7,788$ & NT $\$ 13,790$ & NT $\$ 12,261$ \\
\hline
\end{tabular}

\footnotetext{
${ }^{4}$ In Taiwan, the securities transaction tax is $0.1 \%$ for ETF and the traditional commission fee is $0.1425 \%$ for buying or selling a share.
} 
Table 2 presents the performance of the stacking technique, and Figure 2 shows the timing of a long or short signal obtained from the stacking technique. Accordingly, the total return and percent profitable of stacking are the best in comparison with the four base-learners, even though the prediction accuracy is not the highest. Moreover, the stacking technique can earn a profit return of $19.36 \%$ over the testing period - that is, the only machine learning algorithm used in this paper is able to achieve a positive return. With respect to the uncertainty of performance, the MDD of the stacking technique is much lower than those of base-learners. This also confirms that the stacking technique would outperform single classifiers and homogeneous ensemble classifiers. In addition, as shown in Figure 2, the stacking technique can detect upward and downward trends very fine, although it cannot exactly provide long and short signals at the local trough and local peak, respectively.

Table 2: Profitability comparisons of the four trading rules

\begin{tabular}{lllll}
\hline Trading rule & Stacking & MA20 & MA60 & Buy-and-Hold \\
\hline Prediction accuracy & $66.00 \%$ & - & - & - \\
Number of trades & 31 & 56 & 39 & - \\
Total return & $19.36 \%$ & $-27.00 \%$ & $-20.63 \%$ & $11.10 \%$ \\
Average return & $0.57 \%$ & $-0.56 \%$ & $-0.59 \%$ & - \\
Percent profitable & $45.16 \%$ & $25.00 \%$ & $12.82 \%$ & - \\
Maximum drawdown & NT $\$ 5,463$ & NT $\$ 9,638$ & NT $\$ 32,067$ & - \\
\hline
\end{tabular}

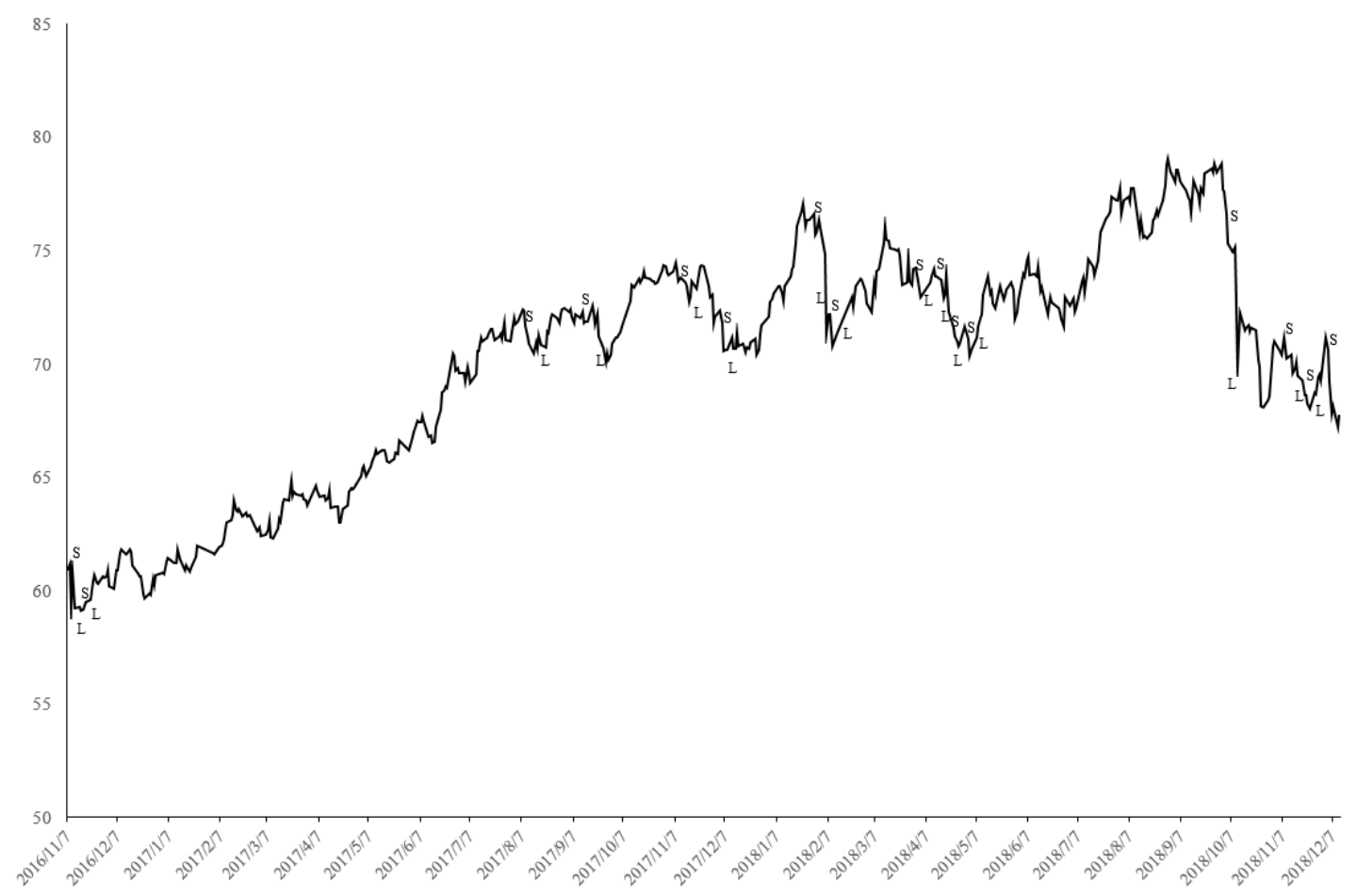

Figure 2: The timing of long and short signals made by the stacking technique Note: $\mathrm{S}$ and $\mathrm{L}$ denote short and long trading signals, respectively. 
Table 2 further compares three commonly-used trading rules: moving-average and buy-and-hold strategies. For moving average strategies, a long (short) signal is determined when the price crosses above (below) the 20-day or 60-day moving average. MA20 and MA60 trading rules suffer negative total returns of $-27.00 \%$ and $-20.63 \%$, respectively. Both moving-average rules generate a lower win rate (percent profitable) than the four base-learners shown in Table 1. We conclude that the performances of moving-average rules are similar to base-learners in that all these strategies are not profitable during the testing period. Smith et al. (2016) argue that technical analysis is useful but depends on different states. They find that the profitability of technical analysis increases during high-sentiment periods. Hence, it might be a possible explanation why moving-average rules are unprofitable in our study. Obviously, the stacking technique can be a better trend indicator in comparison with the moving-average indicator. Finally, although the stacking technique generates 31 trades and produces a lot of trading costs, this technique still beats the buy-and-hold strategy.

\section{Conclusions}

This paper provides a trend-following investing strategy via a two-layer stacking technique, which is a novel ensemble learning approach that combines four famous machine learning algorithms to obtain a better predictor of the trends of stock prices. Using data of the Taiwan Top $50 \mathrm{ETF}$, the results reveal that the stacking technique can generate positive returns with the highest profit, the highest win rate, and the lowest maximum drawdown in comparison with single machine learning classifiers and the conventional moving-average crossover strategy. Most studies in the literature emphasize the high prediction accuracy determined by their approaches, but our findings indicate that high prediction accuracy (such as Adaboost and Xgtoost algorithms) do not ensure better profitability. The proposed two-layer stacking technique is absolutely not the best model for a trend indicator, but we try to provide a new perspective that brings the ensemble learning approach into the set-up of an investing strategy.

\section{References}

Ballings, M., D. Van den Poel, N. Hespeels, and R. Gryp (2015), 'Evaluating Multiple Classifiers for Stock Price Direction Prediction', Expert Systems with Applications, 42, 7046-7056.

Chen, S.S. (2009), 'Predicting the Bear Stock Market: Macroeconomic Variables as Leading Indicators', Journal of Banking \& Finance, 33, 211-223.

Clare, A., J. Seaton, P.N. Smith, and S. Thomas (2016), 'The Trend is Our Friend: Risk Parity, Momentum and Trend Following in Global Asset Allocation', Journal of Behavioral and Experimental Finance, 9, 63-80.

Colombo, E., G. Forte, and R. Rossignoli (2019), 'Carry Trade Returns with Support Vector Machines', International Review of Finance, 19, 483-504.

Duong, T.V.P., S.H. Lin, H.H. Lai, and T.P. Chang (2021), 'Macroeconomic Variables for Predicting Bear Stock Markets of Taiwan and China', International Journal of Emerging Markets, Forthcoming.

Faber, M.T. (2007), 'A Quantitative Approach to Tactical Asset Allocation', The Journal of Wealth Management, 9, 69-79.

Henrique, B.M., V.A. Sobreiro, and H. Kimura (2019), 'Literature Review: Machine Learning Techniques Applied to Financial Market Prediction', Expert Systems with Applications, 124, 226-251.

Huang, W., Y. Nakamori, and S.Y. Wang (2005), 'Forecasting Stock Market Movement Direction with Support Vector Machine', Computers \& Operations Research, 32, 2513-2522.

Hurst, B., Y.H. Ooi, and L.H. Pedersen (2017), 'A Century of Evidence on Trend-Following Investing', The Journal of Portfolio Management, 44, 15-29.

Levine, A., L.H. Pedersen (2016), 'Which Trend is Your Friend', Financial Analysts Journal, 72, 51-66. Menkhoff, L. (2010), 'The Use of Technical Analysis by Fund Managers: International 
Evidence', Journal of Banking \& Finance, 34, 2573-2586.

Neely, C. J., R.D. Rapach, J. Tu, and G. Zhou (2014), 'Forecasting the Equity Risk Premium: The Role of Technical Indicators', Management Science, 60, 1772-1791.

Nyberg, H. (2013), 'Predicting Bear and Bull Stock Markets with Dynamic Binary Time Series Models', Journal of Banking \& Finance, 37, 3351-3363.

Nti, I.K., A.F. Adekoya, and B.A. Weyori (2020), 'A Comprehensive Evaluation of Ensemble Learning for Stock-Market Prediction', Journal of Big Data, 7, 1-40.

Papadamou, S., and S. Tsopoglou (2001), 'Investigating the Profitability of Technical Analysis Systems on Foreign Exchange Markets', Managerial Finance, 27, 63-78.

Raj, M., and D. Thurston (1996), 'Effectiveness of Simple Technical Trading Rules in the Hong Kong Futures Markets'. Applied Economics Letters, 3, 33-36.

Smith, D. M., N. Wang, Y. Wang, and E.J. Zychowicz (2016), 'Sentiment and the Effectiveness of Technical Analysis: Evidence from the Hedge Fund Industry', Journal of Financial and Quantitative Analysis, 51, 1991-2013.

Tsai, C.F., Y.C. Lin, D.C. Yen, and Y.M. Chen (2011), 'Predicting Stock Returns by Classifier Ensembles', Applied Soft Computing, 11, 2452-2459. 